\title{
Designing Evaluation into Open-ended Design Labs
}

\author{
T.C. Muench, P.Eng., \\ Associate Professor of Engineering \\ Listwin Family Chair in Innovative Teaching \\ University of Saskatchewan \\ tim.muench@usask.ca
}

\author{
T. Fonstad, P.Eng., \\ Associate Professor of Engineering \\ Agriculture and Bioresource Engineering \\ University of Saskatchewan \\ terry.fonstad@usask.ca
}

\begin{abstract}
Open-ended design problems are an important part of engineering education. Balanced with this is the requirement for effective evaluation within a limited amount of time. As with concurrent engineering, parallel development of the components is required in this case, the design lab and its evaluation.

The implemented approach uses the engineering design cycle as a lab development template. To facilitate evaluation, the lab document has been designed to require students to clearly complete and document the engineering design cycle.

This paper looks at the lab development process, evaluation results from a sample of graded labs, and feedback results.
\end{abstract}

\section{Introduction}

The design problem specified in this paper is one of seven discipline-specific design labs given to students in a second term freshman course entitled Introduction to Engineering II.

\subsection{Overview and Specification of Lab}

The implemented approach uses the engineering design cycle as a lab development template. The following design steps were used for the initial development of the lab. Section 2 discusses the process details.

The Identification of Need is to develop a design lab representative of Agriculture and Bioresource Engineering, for the 'Introduction to Engineering II' course.

The Problem Definition requires that the lab be an open-ended design (consistent with CEAB definition of Design), and that it includes an effective evaluation strategy.

The Search involved the investigation of previous labs ranging from very open-ended but difficult to evaluate, to limited freedom of design but straightforward evaluation. New ideas were considered, to optimize the balance between openended design and effective evaluation.

The Constraints associated with the lab design are: allotted time (30 minute lecture / 3 hour lab); openended design (constraints can be designed in, to limit the degree); limited student background knowledge (students are beginning second term, freshman year); and suitability for implementation of the formal engineering design cycle (course focuses on engineering design and the design cycle).

The Criteria used to select the best lab are: a relatively open-ended problem (encourage creativity in student designs); effective evaluation (design component evaluated through lab reports and $\log$ books $\sim 400$ students, groups of 4); and content representative of the Agricultural and Bioresource Engineering discipline.

Alternative Solutions / Analyses / Decision combined to yield the most suitable of the various lab concepts considered - the design of a continuous process Cherry Pitter.

The Specification / Communication portion of the Lab Design are key to laying out requirements and process that students must adhere to, to allow openended design that can be effectively evaluated. To facilitate evaluation, the lab document has been designed to require students to clearly complete and document the engineering design cycle. Identification of Need, Problem Definition, Constraints and Criteria are specified up front for students, to limit the open-ended scope for the design. A description of the lab and basic background in continuous processes are provided in the 30 minute lecture. Students are required to do their Search prior to the practical lab period. In the lab, practical experimentation is undertaken to understand the Cherry Pitting process. The culmination of the lab covers Alternative Solutions / Analysis / Decision, where design options are considered and compared. Specification / Communication components occur in 
the report where the resulting design is required to be presented in a format that clearly conveys the design process as well as the design intent of the final design.

\subsection{Overview of Paper}

Section 1 of the paper provides an introduction and overview and specification of the design intent for the lab and its related evaluation.

Details on the development and specification of the lab and its evaluation are discussed in Section 2.

Section 3 investigates the evaluation results of a purposeful sample to provide a range of quality of lab reports. Each report was evaluated (individually, blind study) by 2 faculty members and 2 teaching assistants, using a common grading scheme. Consistency of evaluation and time commitment for the evaluation component of the lab are discussed.

Section 4 looks at the related lab survey statistics and some typical and interesting comments that were provided by students.

Conclusions are discussed in Section 5.

\section{Design of the Lab and its Evaluation}

The engineering design cycle [1] was used as a template to develop the lab. The following section describes the process that was followed to select the best lab design from a group of potential alternatives.

\subsection{Design of the Lab}

The objective was to provide a venue for first year engineering students to learn the design process.

Students are to be evaluated on their ability to communicate the design process they followed and their final design.

\subsubsection{Need}

The Department of Agricultural and Bioresource Engineering needs to develop an open ended design problem for first year engineering students. Engineering colleges need a method of teaching first year students the design process in a laboratory setting.

\subsubsection{Problem}

1. Exercises involving open ended problems used to teach/demonstrate the design process are difficult to evaluate.

2. Problems involving optimization alone rarely include an open ended problem involving creativity and design.

\subsubsection{Search}

Previous attempts at teaching design have included:
1. Design of a mechanical celery harvesting method (Crowe 2000). This project was open ended with the only restriction being that the design had to eliminate any manual labour involved in celery harvesting.

a. Difficulties occurred in evaluating this laboratory exercise. As there was no correct answer, only a design, Teaching Assistants had difficulty determining a method of evaluating the student's reports.

2. Roberge and Fonstad (2001) attempted to show the interaction of shear with several different areas of Agricultural and Bioresource Engineering. Three problems compared shear calculations/considerations in design involving soil shear forces impacting traction of a large loader, shear in mixing of large scale food processing, and shear along the walls of grain storage structures.

a. This laboratory exercise achieved the objective a demonstrating how shear impacted various design problems but it did not involve any actual design, the design process or optimization.

3. Bak and Fonstad (2003) developed a laboratory exercise requiring students to optimize a commercial baking process through heat and belt speed settings.

a. This laboratory exercise demonstrated the interaction of biology and engineering and required students to optimize a process by considering several parameters simultaneously. The exercise did not involve any design or the design process and did not provide students the ability to use creativity in the optimization.

\subsubsection{Constraints}

1. The laboratory exercise must provide students with some insight into the discipline of Agricultural and Bioresource Engineering, BioSystems Engineering or Agricultural and Biological Engineering.

2. Must to be able to explain the laboratory exercise to the students in less than 30 minutes

3 . The laboratory exercise must take no more than $3-$ 6 hours to complete with additional time for preparation of the final report to be submitted.

4. The laboratory exercise must be suitable for first year, second term engineering students

5. The laboratory exercise must be able to be evaluated by Teaching Assistants from different disciplines and without an agricultural engineering background. 


\subsubsection{Criteria}

1. The exercise should have the potential to challenge the students and be enjoyable.

2. It is preferred to utilize a real world problem

3. The exercise should require/inspire creativity using an open ended problem that requires optimization.

4. The problem should be relevant to Saskatchewan or Western Canada.

5. The problem should be relevant to teaching and research at the Department of Agricultural and Bioresource Engineering at the University of Saskatchewan.

6. Students from all backgrounds should be able to relate to the problem.

Each of the criteria was assigned a weighting value, which was used to weight its relative importance.

Table 1 - Criteria Weighting

\begin{tabular}{|c|c|}
\hline Criteria & Weight \\
\hline 1 & 10 \\
\hline 2 & 10 \\
\hline 3 & 30 \\
\hline 4 & 15 \\
\hline 5 & 25 \\
\hline 6 & 10 \\
\hline Total & 100 \\
\hline
\end{tabular}

\subsubsection{Solutions/Analysis/Decision}

1. Reconditioning of the previous celery harvesting laboratory exercise (Crowe 2000)

a. The problem would consist of reducing manual labour during process of celery harvesting and providing as much automation as possible to produce an optimum harvesting system.

2. Energy reduction during cereal grain harvesting.

a. The problem would consist of attempting to reduce energy consumption during cereal grain harvesting.

b. Options could include such things as design of a low energy harvester, extending the harvest period through a method of high moisture harvesting or harvesting the material rapidly combined with secondary processing in a controlled environment over an extended period of time.

3. Design of a moderate capacity cherry pitter for Western Canadian fruit growers.

a. The problem would involve design of a continuous flow cherry pitter capable of processing $500 \mathrm{~kg}$ of cherries per hour.

4. Feasibility study for water supply and wastewater utilization for large scale agri-business development near Saskatoon. a. The problem would involve determining the optimum method/option for water supply and waste water utilization/disposal for development of agri-business animal and grain processing in an area 8 miles east of Saskatoon.

Table 2 indicates that Solution No. 3, Design of a Cherry Pitter, is the best solution of the four analysed.

Solution No. 1 was a not a problem indicated by an industrial partner, was not a Western Canadian problem and was not relevant to teaching and research in the department of Agricultural and Bioresource Engineering.

Solution No. 2 ranked second in our method but there was concern that the problem was too involved and not all students had knowledge of cereal grain harvesting or could learn enough in the period of time involved.

Solution No. 4 did not involve a sufficient design component and required more knowledge and experience than could be expected of first year engineering students.

As such Solution No. 3 was chosen for development of a laboratory exercise for the Department of Agricultural and Bioresource Engineering at the University of Saskatchewan.

Table 2 - Ranking of solutions

\begin{tabular}{|c|c|c|c|c|c|c|c|c|c|}
\hline & & \multicolumn{2}{|c|}{ Sol'n 1} & \multicolumn{2}{|c|}{ Sol'n 2} & \multicolumn{2}{|c|}{ Sol'n 3} & \multicolumn{2}{|c|}{ Sol'n 4} \\
\hline 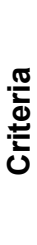 & $\frac{\text { 듬 }}{\frac{0}{0}}$ & 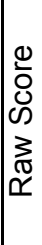 & 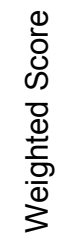 & 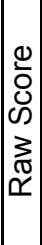 & 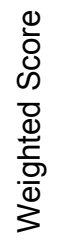 & 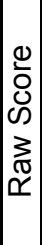 & 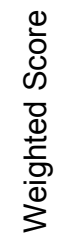 & 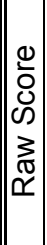 & 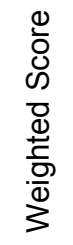 \\
\hline 1 & 0.1 & 4 & 0.4 & 3 & 0.3 & 4 & 0.4 & 3 & 0.3 \\
\hline 2 & 0.1 & 1 & 0.1 & 2 & 0.2 & 4 & 0.4 & 3 & 0.3 \\
\hline 3 & 0.3 & 4 & 1.2 & 3 & 0.9 & 4 & 1.2 & 2 & 0.6 \\
\hline 4 & 0.15 & 1 & 0.15 & 4 & 0.6 & 3 & 0.45 & 4 & 0.6 \\
\hline 5 & 0.25 & 1 & 0.25 & 4 & 1 & 4 & 1 & 3 & 0.75 \\
\hline 6 & 0.1 & 4 & 0.4 & 1 & 0.1 & 4 & 0.4 & 1 & 0.1 \\
\hline & Total & & 2.5 & & 3.1 & & 3.85 & & 2.65 \\
\hline
\end{tabular}

\subsubsection{Specification/Communication}

The Lab handout provides an overview of the need for an automated, continuous flow, medium capacity $(500 \mathrm{~kg} / \mathrm{hr})$ cherry pitter at a cost of $<\$ 10,000$, and the desirability to retain whole cherries (worth twice as much as damaged ones).

An equation was provided for the calculation of puncture force for the cherries, so that the work to pit a cherry could be estimated. An experimental force 
vs. distance plot was obtained from the experimental measurement of the pitting of a cherry using a penetrometer. The area under the curve was determined to provide a numeric value for the energy to pit one cherry $(0.64 \mathrm{~J})$.

Typical cherry properties and dimensions were also provided, some of which are shown in Figure 1.

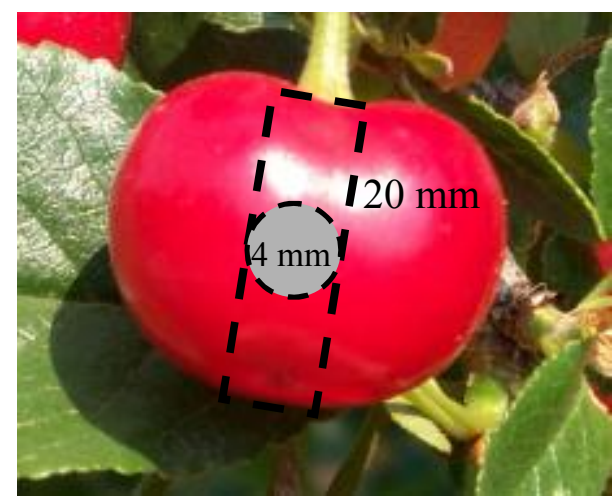

Figure 1 - Cherry properties

Some hand-held cherry pitters, shown in Figure 2, were provided in the lab for the students to examine and use. This allowed students to see the type of tooling used, and to observe the pitting process to see how much damage each process did to the cherries.
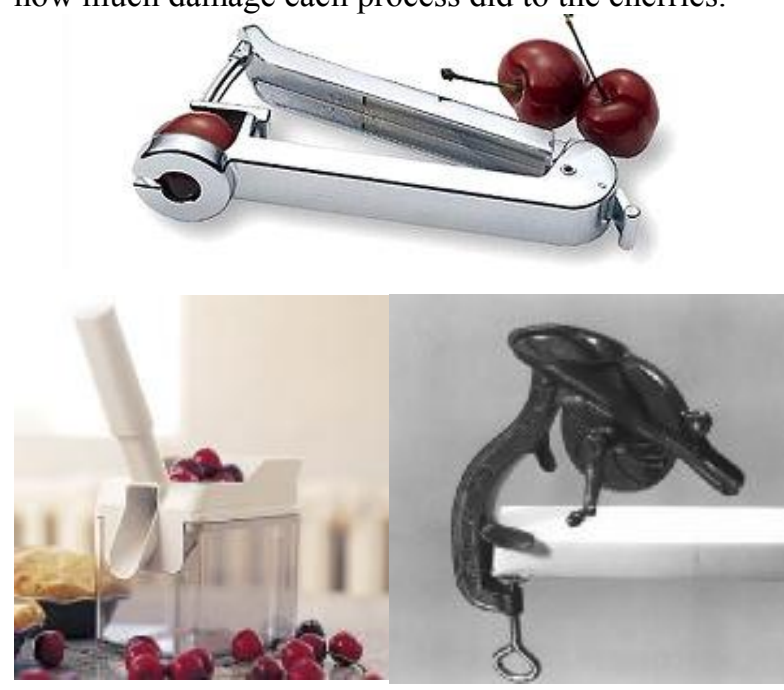

Figure 2 - Manual cherry pitters

Some lecture provided in class gave a cursory overview of continuous processes.

Groups were strongly encouraged to use the resources of the Internet to locate information about cherry pitting and to investigate drive mechanisms and various other components related to processing of fruits and related mechanization.

\subsection{Design of the Evaluation}

The following sections discuss the evaluation methodology, a major component of which is to enforce the design process. In evaluation, normally half the battle (time) is spent searching for the key bits of information on which the evaluation is based.

The layout of the report was modified from the typical technical report format, into a format that reflected the steps of the Design Cycle. The purpose was twofold. First, it forced the students to consciously adhere to the Design Cycle, and secondly, it organized the information in a format that was easy to locate the key components for evaluation.

\subsubsection{Objectives to be Evaluated}

The course in which this lab is presented, teaches the Engineering Design Cycle. In addition, one of the big challenges for budding designers is to convey design intent. Both of these components are required for good design, and as a result, they can be used as an effective indirect measure of a good open-ended design.

The following excerpts from the lab document specify the general marking philosophy and the deliverables.

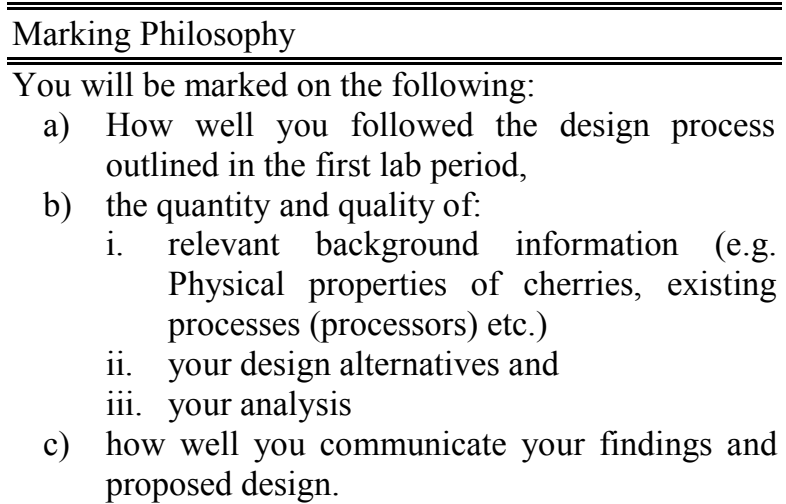

NOTE: You will not be marked on your specific design but rather your ability to follow the design process (which should produce a good design) and communicate your findings to the marker.

\begin{tabular}{l}
\hline \hline Deliverables \\
\hline \hline Your report should be written as a design report to a \\
supervisor (your lab instructor). \\
For this lab only use the design steps as headings. \\
Detailed design drawings are not necessarily \\
required BUT you must somehow communicate \\
your final design to the marker. You can produce \\
AutoCAD, SolidWorks or hand drawn drawings or \\
prototype your final design.
\end{tabular}


Provide an estimate of the power requirement of your design.

BONUS Marks - Environmental (15\%)

Marks will be added to your mark to a maximum of full marks for the lab.

Environmental concerns have been raised by the fact that the juice produced during pitting may not be fit for human consumption due to contact with lubricated machine parts. Additionally, the pits are commonly disposed of in local landfills.

Recommend a method to utilize the cherry pits.

\subsubsection{Weighting of Objectives}

A successful open-ended design, as indicated previously, should be the natural successful conclusion if two conditions are met:

1. The design process is properly implemented.

2 . The design intent is properly communicated.

After much discussion, the weighting of the evaluation was set at:

\section{$60 \%$ - Design Process}

$40 \%$ - Design Communication

This weighting was further refined in the marking key that was provided to the students.

\subsubsection{Marking Key for Students}

The marking key shown in Table 3 was provided to students as part of the Lab document.

Table 3 - Marking key for students

\begin{tabular}{||c||c||}
\hline Point & Section \\
\hline \hline 1 & Need and problem definition \\
\hline \hline 1 & Literature review (search) \\
\hline \hline 1 & Constraints \\
\hline \hline 1 & Criteria \\
\hline \hline 1 & Analysis (must use the criteria) \\
\hline \hline 4 & $\begin{array}{c}\text { Communication of the Design } \\
\text { (decision, specification, } \\
\text { communication) }\end{array}$ \\
\hline
\end{tabular}

\section{Evaluation Results and Statistics}

To determine the effectiveness of evaluating this open-ended lab, data was collected from multiple evaluations of a sample of the lab reports. The following section details the method and results.

\subsection{Experimental Method}

To gain insight into the ability of the evaluation to meet the goals set out, a sample of labs was collected and independently evaluated multiple times.

\subsubsection{Sampling Method and Sample Size}

A purposeful sample of labs was collected to cover a cross-section of lab report quality. Due to time constraints, the sample was limited to 10 lab reports.

\subsubsection{Evaluation Method}

The sample of 10 lab reports was submitted for 4 independent evaluations. Two faculty members and 2 Teaching Assistants were provided a marking key, and asked to grade the reports, and to record the amount of time spent evaluating each of the lab reports.

\subsection{Evaluation Results}

Although data were recorded for each individual point in the evaluation, statistics were compiled only for the final grade of each lab report, and the amount of time required to evaluate it.

\subsubsection{Means and Standard Deviations}

Table 4 - Lab Evaluation Statistics

\begin{tabular}{|c|c|c|c|c|c|}
\hline Lab 1 & Mean & $\begin{array}{l}\text { Std } \\
\text { Dev }\end{array}$ & Lab 6 & Mean & $\begin{array}{l}\text { Std } \\
\text { Dev } \\
\end{array}$ \\
\hline Total & 8.33 & 1.16 & Total & 6.74 & 0.65 \\
\hline Profs & 7.40 & 0.71 & Profs & 6.73 & 0.39 \\
\hline TAs & 9.25 & 0.35 & TAs & 6.75 & 1.06 \\
\hline Lab 2 & Mean & $\begin{array}{l}\text { Std } \\
\text { Dev } \\
\end{array}$ & Lab 7 & Mean & $\begin{array}{l}\text { Std } \\
\text { Dev }\end{array}$ \\
\hline Total & 7.75 & 0.52 & Total & 5.31 & 1.01 \\
\hline Profs & 7.88 & 0.67 & Profs & 5.13 & 0.95 \\
\hline TAs & 7.50 & $\mathrm{~N} / \mathrm{A}$ & TAs & 5.50 & 1.41 \\
\hline Lab 3 & Mean & $\begin{array}{l}\text { Std } \\
\text { Dev } \\
\end{array}$ & Lab 8 & Mean & $\begin{array}{l}\text { Std } \\
\text { Dev }\end{array}$ \\
\hline Total & 7.13 & 1.13 & Total & 5.96 & 0.99 \\
\hline Profs & 7.85 & 0.35 & Profs & 6.55 & 0.21 \\
\hline TAs & 6.40 & 1.27 & TAs & 5.38 & 1.24 \\
\hline Lab 4 & Mean & $\begin{array}{l}\text { Std } \\
\text { Dev } \\
\end{array}$ & Lab 9 & Mean & $\begin{array}{l}\text { Std } \\
\text { Dev }\end{array}$ \\
\hline Total & 9.59 & 0.12 & Total & 4.65 & 1.58 \\
\hline Profs & 9.55 & 0.07 & Profs & 4.18 & 2.02 \\
\hline TAs & 9.63 & 0.18 & TAs & 5.13 & 1.59 \\
\hline Lab 5 & Mean & $\begin{array}{l}\text { Std } \\
\text { Dev } \\
\end{array}$ & $\begin{array}{l}\text { Lab } \\
10 \\
\end{array}$ & Mean & $\begin{array}{l}\text { Std } \\
\text { Dev }\end{array}$ \\
\hline Total & 4.24 & 1.57 & Total & 6.71 & 1.22 \\
\hline Profs & 3.23 & 0.39 & Profs & 5.93 & 0.04 \\
\hline TAs & 5.25 & 1.77 & TAs & 7.50 & 1.41 \\
\hline
\end{tabular}


Means and Standard Deviations were reported for the total sample. Observations of the initial data showed some trends in Faculty vs. Teaching Assistant (TA) results, so the data was further processed by splitting the data into these 2 groups. All results are shown in Figure 4.

\subsubsection{Average Means and Standard Deviations}

Due to the small samples (4 per lab, or 2 per lab when divided into faculty vs. TAs), individual lab results do not have great statistical power. To improve the statistical power, averages were computed to determine trends of the results. The results are shown in Table 5.

Table 5 - Average statistics

\begin{tabular}{||c|c|c||}
\hline \hline \multicolumn{3}{|c|}{ Averages } \\
\hline \hline & Mean & Std Dev \\
\hline \hline Total & 6.64 & 1.00 \\
\hline Profs & 6.44 & 0.58 \\
\hline TAs & 6.83 & 1.14 \\
\hline
\end{tabular}

3.2.3 Evaluation - Faculty vs. Teaching Assistant Examination of the average statistics in Table 5 shows the following:

An average mean, although not normally meaningful, shows that TAs provided grades that were approximately 0.4 marks higher, out of the 10 mark total for the lab reports.

The average Standard Deviation for the lab reports graded by the faculty members was about half that of the TAs.

The overall average Standard Deviation was 1 mark out of 10 , which confirms that the grading was relatively consistent.

\subsubsection{Time Required per Evaluation}

Average times varied significantly between faculty and TAs as shown in Table 6. Although statistical analysis was not conducted for correlation, it is likely that the longer time spent by faculty resulted in the lower standard deviation of marks by faculty members that was shown previously in Table 5 .

Table 6 - Average time to evaluate labs

\begin{tabular}{||c|c||c||}
\hline \multicolumn{2}{|c||}{ Average Time to Evaluate Lab } \\
\hline \hline Total & 9.31 & \\
\hline Faculty & 11.30 & Faculty minus TA \\
\hline TAs & 6.90 & 4.40 \\
\hline \hline
\end{tabular}

\subsection{Observations - Design Success / Challenges}

In addition to the hard data of the evaluation, some anecdotal information is provided on the successes and challenges of the designs that were submitted. It was obvious that a vast majority of groups spent a lot of time thinking the designs through in detail. The following sections describe some general trends that were observed.

\subsubsection{Creating Order}

The first step in the automation of cherry pitting is to create order so that the cherries can be accurately located for the pitting process. There were many good and innovative ideas, including floating the cherries into dividers, and using various hoppers and wipers to locate the cherries. Although many were well thought out, many others relied on mechanical 'chance' for alignment, and assumed things would fall correctly into place, and would not jam (excuse the pun!).

\subsubsection{Pitting Operation}

Numerous solutions were proposed to the physical pitting, some of which were much more plausible than others. Most used the proven mechanical method, but many options were proposed including water jets, and using the momentum of airborne cherries as a twist in the mechanical pitting process.

\subsubsection{Mechanization and Synchronization}

In general, most designs showed that significant thought had gone into the process, but there were varying degrees of practicality in the implementation of the mechanization, due to the limited background knowledge in this area. There were a number of innovative solutions, including double acting strokes.

There was also a lack of appreciation of synchronization between the cherries and the pitting devices. Issues such as the arc trajectory of a pitter shaft on a drum approaching and piercing a cherry were not considered in adequate detail. There also seemed to be a general under-estimation of mechanical cycle times.

\subsubsection{Communicating the Design}

Most groups communicated their designs using sketches. Students received sketching instruction in the previous semester, and were quite successful with this method.

A few groups created CAD models of their final designs, and those that did created 3D CAD models and presented isometric or rendered images.

One group submitted a hand made physical prototype. 


\subsubsection{Overall Quality}

Based on the technical level of the students, the scope of the project, and the limited amount of time, the results were very encouraging.

\section{Student Feedback and Comments}

A student survey was conducted to determine their reactions to the lab and their opinions on how it affected their design skills.

\subsection{Student Survey Feedback}

A voluntary survey was conducted to acquire student feedback on the lab. Part 1 was designed as a categorical response to indicate the degree of agreement with statements related to the lab, or the students perception of the lab. The response options were:
1. Strongly Agree
4. Somewhat Disagree
2. Agree
5. Disagree
3. Somewhat Agree
6. Strongly Disagree

\subsubsection{Sample Size}

Although participation was voluntary, 221 surveys were returned, which is significantly over half of all the registered students

\subsubsection{Handling of Incomplete Records}

Question were independent, so responses were still used when a student's overall record was incomplete.

\subsubsection{Results}

For each of the questions that follow in Figures 3-9, a detailed distribution of student responses is shown, followed by a Total of Agreement responses versus Total of Disagreement responses.

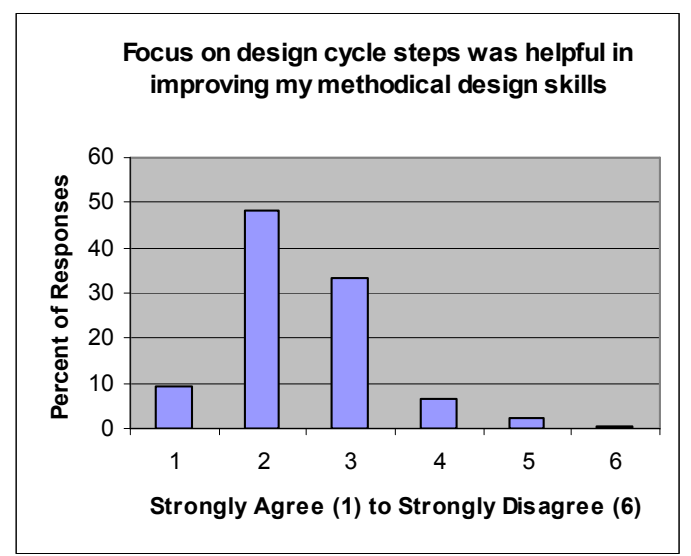

Figure 3 - Improved design skills

Agreement - 91\% Disagreement - 9\%

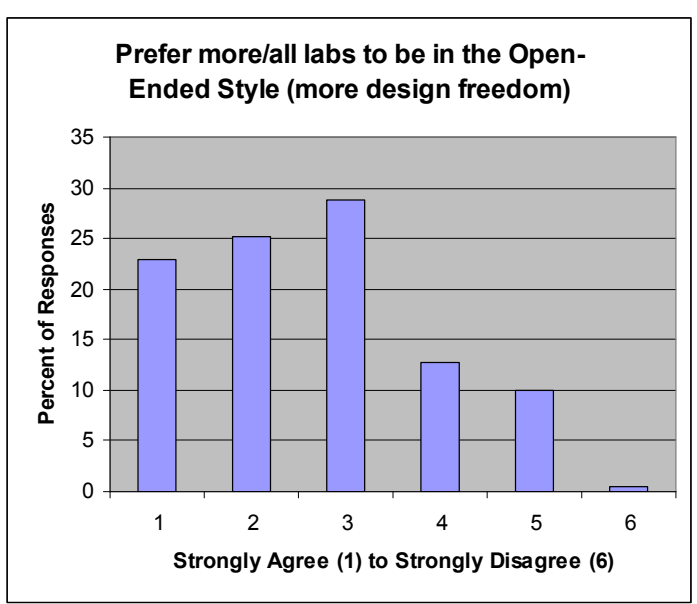

Figure 4 - Prefer open-ended labs

Agreement - 77\%

Disagreement - 23\%

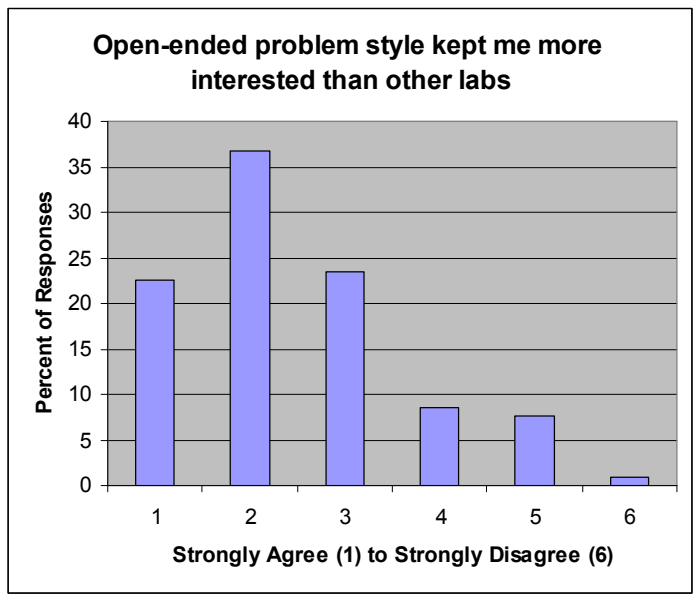

Figure 5 - Open-ended more interesting Agreement - 83\%

Disagreement - 17\%

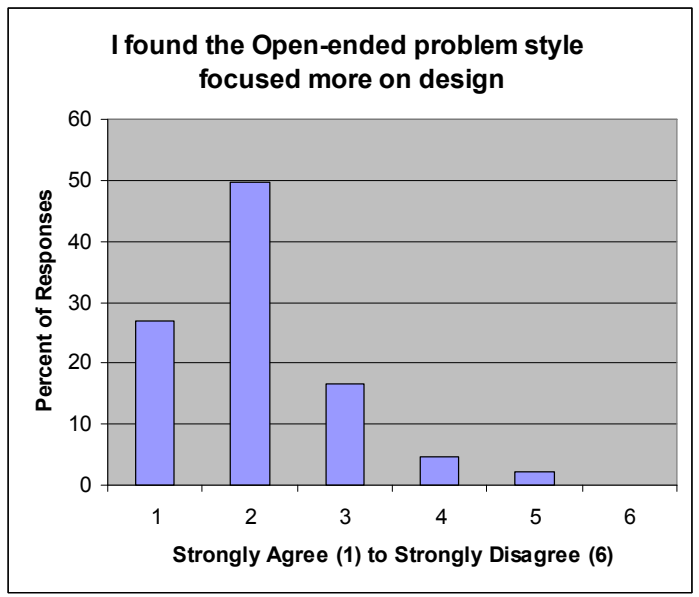

Figure 6 - Open-ended focused on design Agreement - 93\%

Disagreement - 7\% 


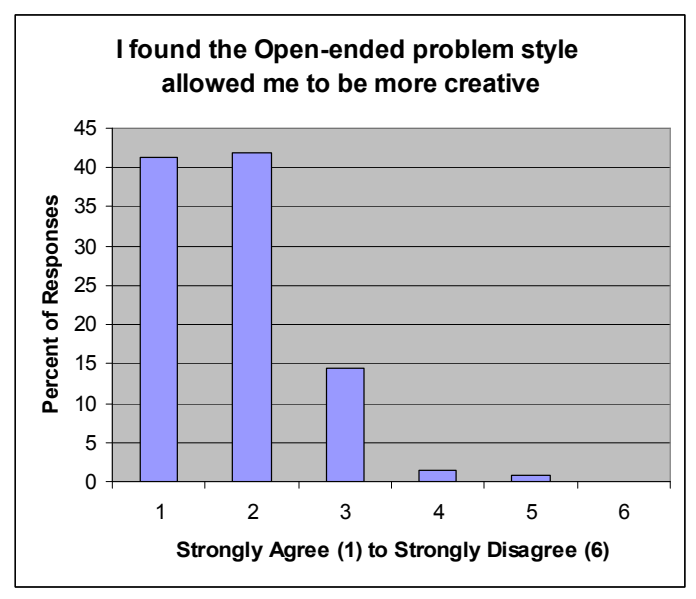

Figure 7 - Open-ended more creative Agreement - 98\%

Disagreement - 2\%

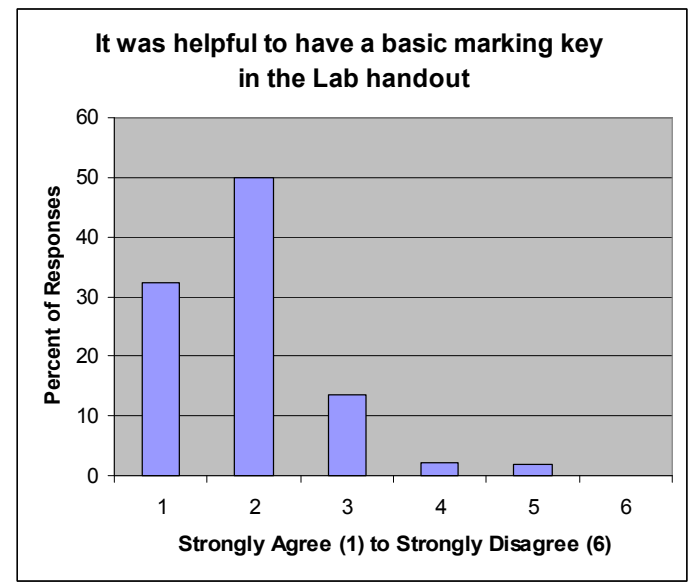

Figure 8 - Marking key helpful

\section{Agreement - 96\% Disagreement - 4\%}

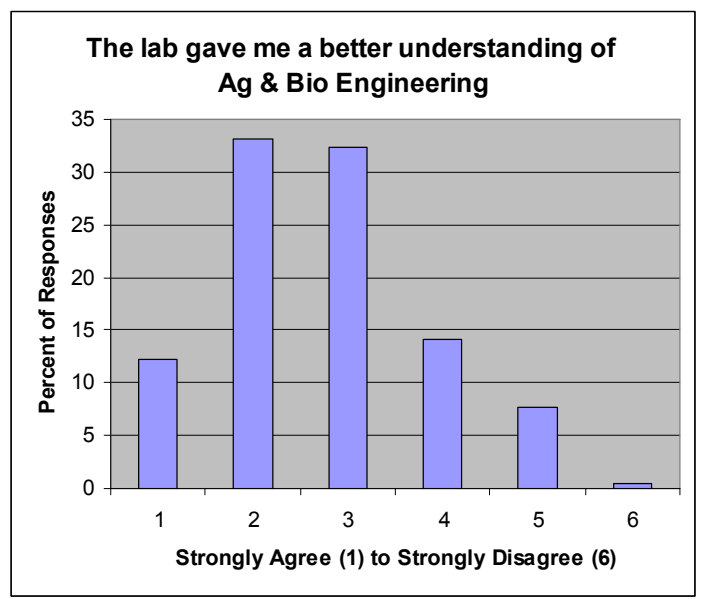

Figure 9 - Better understanding of Ag \&Bio Eng'g Agreement - 78\%

Disagreement - $22 \%$

\subsubsection{Summary of Results}

The responses to the four questions listed below were overwhelmingly in agreement, with a minimum of $90 \%$ of the responses in agreement, or 9 of 10 respondents.

The focus on design cycle steps was helpful in improving my methodical design skills

I found that the open-ended problem style focused more on design

I found that the open-ended design style allowed me to be more creative

It was helpful to have a basic marking key in the lab handout

The remaining three questions that follow were still very strongly in agreement, at approximately $80 \%$, or 4 of 5 respondents.

I prefer morelall of the labs to be in the open-ended style, which allows more design freedom

The open-ended problem style kept me more interested than the other labs

The lab gave me a better understanding of Agricultural and Bioresource Engineering

\subsection{Student Comments}

Two questions were designed to elicit responses from students, to allow more freedom of response.

The two questions were:

What I enjoyed most about the lab

What I found most difficult about the lab

The number of written responses to the questions were 160 and 156 respectively. Responses were examined for common themes and were then categorized according to the common themes. The results are shown in Figures 10 and 11 respectively.

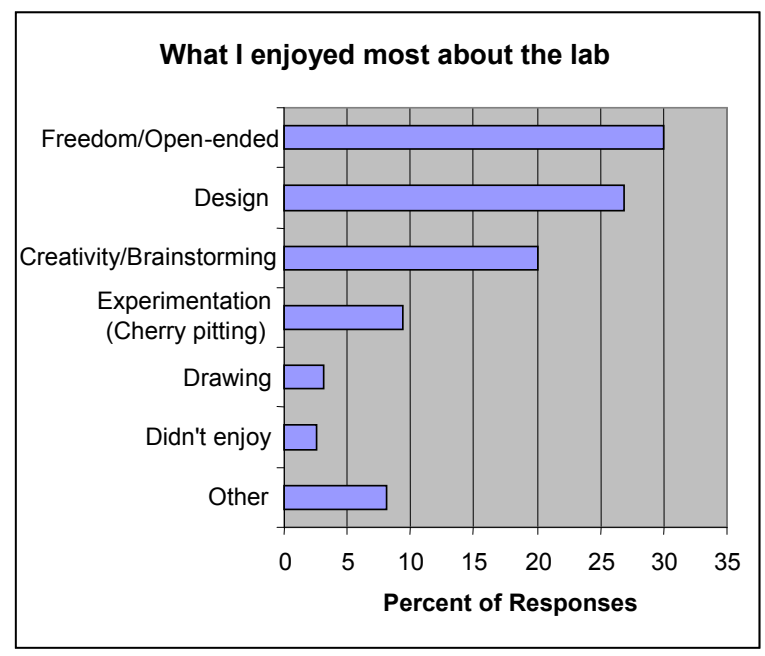

Figure 10 - Enjoyed most about lab 


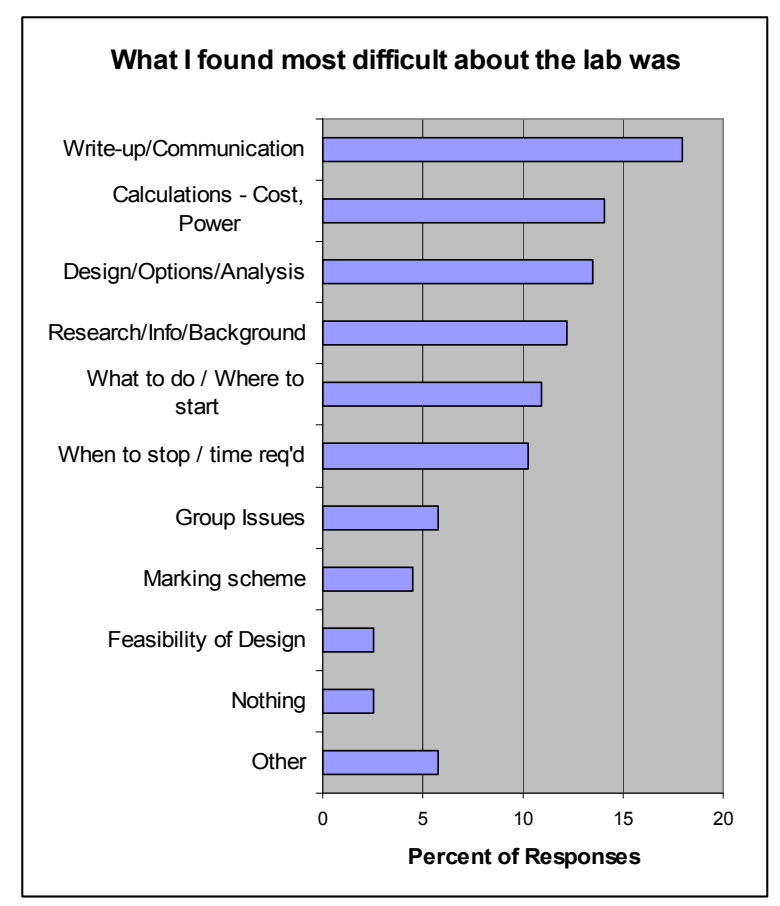

Figure 11 - Most difficult about lab

\subsubsection{General Trends and Recurring Themes}

The major enjoyable aspects of the lab are summarized by Freedom/Open-ended, Design and Creativity. All of these are characteristics of an openended design.

By far, the majority of the comments on the "most difficult' portion of the lab were related to the lack of related experience in both technical background, and in tackling open-ended problems. These were expected with the background of the students, but future iterations of such a lab may focus more on providing a bit more relevant background.

\subsubsection{Interesting Comments}

A response to the 'most difficult part of the lab'

"Nothing - it was enjoyably difficult"

A number of responses had the 'most enjoyable' aspect AND the 'most difficult' aspect identical

"Designing the pitting machine"

"Coming up with different designs"

meaning that the things that made it difficult for some, made it also enjoyable.

\section{Conclusions}

The objective of this work was to illuminate a method for successfully designing evaluation into open-ended design labs. To this end, a number of conclusions follow.

\subsection{Design of the Evaluation}

Adhering to the engineering design cycle and communicating design intent are key components in ensuring a successful open-ended engineering design.

These 2 key components can be used as an effective method of indirectly evaluating open-ended designs.

\subsection{Evaluation Results}

The objectives for consistency of evaluation and efficiency (measured by time commitment per lab) of evaluation were achieved.

Average standard deviation for lab report grades was 1 (out of a score of 10).

Average grading time per lab was just over 9 minutes.

Teaching Assistants took almost 4.5 minutes less per lab to grade, which may explain why they also had a standard deviation almost twice as large.

Teaching Assistants provided a grade almost 0.5 higher than faculty, on average. This may be due to providing the 'benefit of the doubt' since they have recently been on the receiving end of the grading process.

\subsection{Student Feedback}

Student feedback was overwhelmingly positive. Questions related to: The focus on design cycle steps, open-ended problem design, open-ended creativity, and the helpfulness of a marking key

were all supported by agreement of 9 out of 10 students.

Questions related to: Morelall of the labs to be in the open-ended style, open-ended problem being more interesting, and lab giving a better understanding of Agricultural and Bioresource Engineering

were all supported by agreement of 4 out of 5 students. Student comments on the most enjoyable part of the lab focused on freedom of open-ended problems, design, and creativity. Comments on the most difficult part of the lab were related to the lack of related experience in both technical background, and in tackling open-ended problems. These were expected with the background of the students, but future iterations of such a lab may focus more on providing a bit more relevant background.

\section{References}

[1] Eide, A.R., D.J. Jenison, L.H. Mashaw, and L.L. Northrup, Engineering Fundamentals and Problem Solving - Fourth Edition, McGraw Hill, pp. 80-81 